\title{
Der Tonus peregrinus in der Theorie und Praxis. Eine Gegenüberstellung von spätmittelalterlicher musiktheoretischer Kompendien und Choralhandschriften
}

\section{The Tonus peregrinus in Theory and Praxis. Comparing Evidences of Late Medieval Theoretical Treatises and Chant Books}

\author{
Ágnes Papp / papp.agnes@btk.mta.hu \\ Hungarian Academy of Sciences, Research Centre for the Humanities, Institute \\ for Musicology, Department of Early Music History, Budapest, HU
}

\begin{abstract}
The history of tonus peregrinus has been treated in literature in many different ways. Covering the early phase of the term "peregrinus" has given an opportunity to examine the archaic modalities within the Gregorian antiphon repertory. In the 9th-10th centuries, when terms and examples of the "neophytus tonus" or "parapteres" and "tonus novissimus" respectively were put down in writing, the practice of psalmody and the inclusion of the melodies into the system of Octoechos did not follow strictly fixed norms yet. The "differentia peregrina" emerged as a characteristic appellation - as a label of an extraordinary psalm cadence - in the south German music theoretical writings of the late Middle Ages. It rose unnoticed from the irregular ending of the 8th mode to the rank of an independent tone and there are hardly any treatises and tonaries in Central Europe in the 14th-15th-centuries, moreover, following the Middle Ages that would fail to discuss it.

The study intends to show how the notations of this rare melody formula in theoretical writings supplement the data missing from practical chant books. The explanations and antiphon examples of the late treatises included to demonstrate this tone help to elucidate the rational interpretations and musical considerations which contributed to keeping the concept of tonus
\end{abstract}

Der Beitrag wurde von dem Projekt NK 104426 des National Research, Development and Innovation Fund (früher: Ungarischer Förderungsfonds der Wissenschaftlichen Forschung - OTKA) unterstützt. 
peregrinus alive. And last but not least, the antiphons cited are used to examine how the singing practice supported or took into consideration the theoretical systematization.

\section{Key words}

Gregorian chant, psalm tone, theory of the eight modus, music treatises from the Middle Ages, tonary, tonus peregrinus, antiphon

Um die archaischen Modalitäten gut zu verstehen, ist die Erforschung des davon untrennbaren Peregrinus - als Terminus, als Psalmton und als Melodietyp - unerlässlich. Es wurde immer wieder im Zusammenhang mit der Frühphase der Psalmodiepraxis und im Hinblick der Einordnung von Melodien ins System des Octoechos untersucht. ${ }^{1}$ Seit dem frühesten Erscheinen des Begriffs in den theoretischen Traktaten bezeichnete das Adjektiv „peregrinus” diejenige Kadenzformel und Antiphonenmelodien, die sich eben nicht genau in den Rahmen der acht Psalmtöne eingliedern ließen. Modulationsfähige und irreguläre Antiphonen wurden den Kategorien neophytus tonus und parapteres zugeordnet; darunter waren sogar der spezifischen peregrinae Psalmformel zugehörige charakteristische Stücke zu finden, wie z. B. Nos qui vivimus usw. ${ }^{2}$ Außergewöhnliche und überzählige Psalmkadenzen (Differentiae) des 1. Modus waren auch seit dem 11. - 12. Jahrhundert übereinstimmend bis zu der späten theoretischen Überlieferung aus Zentraleuropa in den Tonaren und Traktaten als peregrinae genannt.

„[...] in primo tono aliis quam nos utuntur differentiis ad hanc antiphonam: Biduo vivens, ita: Seculorum amen. Itemque Saeculorum amen, $\mathrm{O}$ beatum pontificem tono videlicet inferius hanc finientes, multaque id genus. In quarto vero in hac antiphona: Nos scientes, in septimo in ista: Loquebantur, peregrinos quosdam modulantur sonos, et si qua inveniri possunt similia." (BERNO Prol. ${ }^{3}$ 12.29-34)

1 Vgl. CLAIRE, Dom Jean. Lés Répertoires Liturgiques Latins Avant Octoechos. Études Grégoriennes, 15. Solesmes: Abbaye Saint-Pierre, 1975, S. 89-92; Idem. The Tonus Peregrinus - A Question Well Put? In Orbis Musicae: Studies in Musicology, 7. Tel Aviv: Department of Musicology, Tel Aviv University, 1980, S. 3-15.; FALCONER, Keith. The Modes before the Modes: Antiphon and Differentia in Western Chant. In The Study of Medieval Chant. Paths and Bridges, East and West. In Honour of Kenneth Levy. Peter Jeffery (ed.). Woodbridge: The Boydell Press, 2001, S. 131-145.

2 Die besprochenen Quellen sind: Aurelian. und Mod. Autenticus (Pseudo-Hucbald: De modis) Vgl. ATKINSON, Charles M. Parapter. In Handwörterbuch der musikalischen Terminologie, IV. Riethmüller, Albrecht (Hrsg.). Stuttgart: Franz Steiner Verlag, 1978; Idem. The Parapteres: Nothi or Not? Musical Quarterly, 68. 1982, 32-59. (hier: 36.)

3 Die theoretischen Abhandlungen werden bei der Zitierung verkürzt, bzw. mit den Sigeln des Lexicon musicum Latinum medii aevi (LmL) gekennzeichnet. Vgl. BERNHARD, Michael (Hrsg.). Lexicon Musicum Latinum Medii Aevi. Wörterbuch der lateinischen Musikterminologie des Mittelalters bis zum Ausgang des 15. Jahrhunderts, 1. Faszikel, Quellenverzeichnis (2. Auflage). München: Verlag der Bayerischen Akademie der Wissenschaften, 2006.

4 RAUSCH, Alexander. Die Musiktraktate des Abtes Bern von Reichenau. Musica Mediaevalis Europae Occidentalis, Bd. 5. Walter Pass (Hrsg.). Tutzing: Schneider, 1999, S. 67-68. Zitiert wird auch von: BERNHARD, Michael. The Seligenstadt Tonary. Plainsong and Medieval Music, 13. 2004, S. 107-125. (hier: S. 116) 
„Peregrini eiusdem toni. Saeculorum, Amen. Speciosus / Saeculorum, Amen. Biduo vivens.“ (Wolfenbüttel, Herzog August Bibl., Helmst. 1050, fol. 17-27) ${ }^{5}$

„Ille vero peregrinae primi toni differentiae ut: [...] Speciosus forma vel [...] Biduo vivens, $\mathrm{O}$ beatum pontificem penitus abiciendae sunt, quia nullius auctoritatis sunt.“ (TON. Seligenst.) ${ }^{6}$

„Sequuntur differencie primi toni non competentes sive peregrine [...] ut patet in hac antiphona Biduo vivens pendebat [...]“ (LAD. ZALK. 4, 117-118) ${ }^{7}$

Das heißt, dass dieser Begriff im musiktheoretischen Schrifttum nicht ausschließlich mit einem einzigen Modus verbunden war. Trotzdem des Öfteren bezeichnete er die extraordinäre Psalmkadenz des 8. Modus:

„Sciendum est quod usus quartam dat differentiam octavo tono sub qua una tantum cantatur antiphona scilicet Nos qui vivimus et dicitur tonus peregrinus. Explicit de octo tonis." (IAC. TWING. $)^{8}$

„Differentia quarta. Posset et annecti differentia quarta, quam tonum peregrinum appellamus idcirco, quia hunc praefati toni repellunt longius stare a se; huius melodia est [...]“ (MICH. KEINSP.) ${ }^{9}$

Von der zusätzlichen Differenzformel des 8. Modus erreichte die Differentia peregrina laut konkreter theoretischen Abhandlungen und Tonarquellen den Rang eines selbstständigen Psalmtons.

„Quidam addunt hic unam aliam differentiam octavi toni, quam vocant 'tonum peregrinum "“ (GOB. PERS. $)^{10}$

„Et nota: licet sit tonus peregrinus, hic tamen racione finis ad octavum tonum reducitur.“ (TRAD. Holl. XVII 235) ${ }^{11}$

Als eine solche Art vom Terminus technicus taucht der Begriff Tonus peregrinus vor allem in zentraleuropäischen Traktaten auf, die seit dem 14. Jahrhundert eine praktisch

5 Es handelt sich um eine Kopie des karolingischen Tonars. Vgl. HUGLO, Michel. Les Tonaires. Inventaire, Analyse, Comparaison. Paris: Société Française de Musicologie, 1971. S. 35.; Idem. Grundlagen und Ansätze der mittelalterlichen Musiktheorie von der Spätantike bis zur Ottonischen Zeit. In: Die Lehre vom einstimmigen liturgischen Gesang. Geschichte der Musiktheorie, Bd. 4. Thomas Ertelt - Frieder Zaminer (Hrsg.). Darmstadt: Wissenschaftliche Buchgesellschaft, 2000, S. 17-102. (hier: 85.)

6 BERNHARD 2004, op. cit., S. 110.

7 BERNHARD, Michael - WITKOWSKA-ZAREMBA, Elżbieta (Hrsg.). Traditio Iohannis Hollandrini [im Weiteren: TIH], Band VI, Die Traktate XXII - XXVI, Ladislaus de Zalka und Szydlovita, Appendices [...] Bayerische Akademie der Wissenschaften. Veröffentlichungen der Musikhistorischen Komission, Band 24. München: Verlag der Bayerischen Akademie der Wissenschaften, 2015, S. 374.

8 MATHIAS, F. X. (Hrsg.). Der Straszburger Chronist Königshofen als Choralist. Sein Tonarius. Graz: Styria, 1903, 138.

9 KEINSPECK, Michael. Lilium musicae planae. Augsburg, 1500.

10 MÜLLER, Hermann. Der tractatus musicae scientiae des Gobelinus Person. Kirchenmusikalisches Jahrbuch, Jg. 20, 1907, S. 177-196. (hier: S. 196, Sp. b.) - Siehe auch BERNHARD 2004, op. cit., S. 118, Fn. 16.

11 BERNHARD, Michael - WITKOWSKA-ZAREMBA, Elżbieta (Hrsg.). TIH, Band V, Die Traktate XV - XXI. Bayerische Akademie der Wissenschaften. Veröffentlichungen der Musikhistorischen Komission, Band 23. München: Verlag der Bayerischen Akademie der Wissenschaften, 2014, S. 189. 
orientierte Elementarlehre der Musik vermittelten. ${ }^{12}$ Eine beträchtliche Anzahl dieser Traktaten aus dem 15. Jahrhundert kann mit dem Namen von Johannes Hollandrinus als Autorität klar verbunden werden; hiermit gehört der Terminus und die Lehre zu der Hollandrinus-Tradition wie ein locus auxiliaris zu. ${ }^{13}$ Der feste Begriff Tonus peregrinus lässt sich auch in gedruckten musiktheoretischen Lehrbüchern des 16. Jahrhunderts aus dem deutschen Sprachgebiet und aus Ostmitteleuropa wiederfinden. ${ }^{14}$

Die für peregrina eingestufte Psalmkadenz war seit ihren frühesten Dokumenten bis zu den Tonaren der schon erwähnten Hollandrinus-Tradition fortan abwertend bewertet, somit nicht anders angeführt und charakterisiert, als ein etwas überzähliges und unregelmäßiges Anhängsel.

„Differentia octava quasi rara ac barbara in ultimo ponitur extraordinaria [...]“ (BERNO ton. 8.8) ${ }^{15}$ „Illae autem neque competentes neque necessariae sunt, que non in recto tenore sed secundum libitum canentium adaptantur." (IOH. COTT. ton.) ${ }^{16}$

„Dicitur autem peregrina, ideo, quod rara in psalmodia." (HUGO SPECHTSH.) $)^{17}$

„Alie vero differencie sunt non necessarie, ut sunt ille, que non necessitatis causa, set solius curialitatis causa a quibusdam et presertim religiosis asserviuntur, de quibus non necessarium dicere, ut sunt peregrini $[\ldots]^{\text {“. }}$ (TRAD. Holl. VI 42, 14) ${ }^{18}$

Der in den Kompendien der acht Psalmtöne hinzugefügte Tonus peregrinus zeigt sich als ein wohl bekanntes Gebilde, dessen bestimmender Faktor der Tenorwechsel der beiden Vershälften ist. Auch wenn der Gebrauch des Terminus auf eine bestimmte Region und musiktheoretische Tradition beschränkt war, lässt sich die Psalmdifferenz selbst, und auch die ganze Melodieformel, aus einer viel breiteren Quellengrundlage aus gan-

12 Erbacher nannte zahlreiche Autoren als Quellen der musikalischen Gestalt und der Beschreibung des Tonus peregrinus in seiner Monographie: ERBACHER, Rhabanus. Tonus Peregrinus. Aus der Geschichte eines Psalmtons. Münsterschwarzach: Vier-Türme-Verlag, 1971.

13 Vgl. BERNHARD, Michael - WITKOWSKA-ZAREMBA, Elżbieta (Hrsg.). TIH, Band I. Die Lehrtradition des Johannes Hollandrinus. Bayerische Akademie der Wissenschaften. Veröffentlichungen der Musikhistorischen Komission, Band 19. München: Verlag der Bayerischen Akademie der Wissenschaften, 2010, S. 52, 67-68.

14 KEINSPECK, op. cit.; WOLLICK, Nicolaus. Opus aureum. Köln, 1501; COCHLAEUS, Johannes. Tetrachordum musices. Nürnberg, 1514; ORNITOPARCHUS, Andreas. Musice Active Micrologus. Leipzig, 1517; FELSZTYN, Sebastian. Opusculum musicae compilatum. Krakau, 1517; LIBAN, Jerzy. De musicae laudibus oratio. Krakau, 1540; FINCK, Hermann. Practica musica. Wittemberg, 1556.

15 RAUSCH 1999, op. cit., S. 102.

16 SMITS VAN WASBERGHE, Joseph (Hrsg.). Iohannis Affligemensis: De Musica cum Tonario. Corpus Scriptorum de Musica, 1. Rome: American Institute of Musicology, 1950, S. 154.

17 BECK, Carl (Hrsg.). Flores musice omnis cantus gregoriani von Hugo von Reutlingen. Stuttgart: Literarischer Verein, 1868, S. 142.

18 RAUSCH, Alexander (Hrsg.). Opusculum de musica ex traditione Iohannis Hollandrini. A Commentary, Critical Edition and Translation. Ottawa: The Institute of Meidaeval Music, 1997, S. 76. 
zem Europa ermitteln und belegen. ${ }^{19}$ Die Aufzeichnungen der vollen Psalmmelodie in Tonaren, musiktheoretischen Abhandlungen oder in Lehrbüchern nehmen die Stelle der aus der praktischen Choralhandschriften fehlenden Angaben ein.

Die Verwendung des peregrinus Tonus scheint nämlich recht beschränkt zu sein: entweder kam es nur im Zusammenhang mit einigen wenigen Antiphonengesängen vor, (wie bei Aurelianus Reomensis) oder verknüpfte sich mit dem Text des Psalms 113, „In exitu Israel“ ${ }^{\text {“ }}{ }^{20}$ dem Prozessionsgesang der Ostervesper um das Baptisterium. Infolge des geläufigen liturgischen Gebrauchs von der Melodieformel wurde ausschließlich die Bezeichnung „Paschalis“ oder „Tempore Paschali“ in den Tonaren angegeben. Hiermit wurde eine merkliche Verbindung zwischen dem spezifischen Modus und der signifikanten liturgischen Stelle angedeutet. ${ }^{21}$

Die Autoren der späteren Traktate waren sogar zugeneigt, Geltung und Anwendung des Psalmtons auf etliche regionale liturgische Bräuche zu beschränken, und es als eine jüngere, moderne Entwicklung zu betrachten.

„Quidam tenores dicuntur peregrini seu usuales, illi scilicet, quos usuardi quidam et indocti in diversis ecclesiis psalmodiis applicuere finalibus nec principales curantes antiquorum tenores nec curiosos, scilicet artificiales novos. De quibus reputatur tenor ususalis illius psalmi In exitu Israel [...] super antiphonam Nos qui vivimus, qui octavi magis debet dici quam alterius propter antiphonam ex finali octavo debitam." (HEINR. EGER) ${ }^{22}$

„Quarta vero peregrina dicitur, cuius differentia antiphonas antiqui sub principali tono [...] collocarunt. Quo fit, ut adhuc a paucis curatur [...]“ (Wollick: Opus aureum) ${ }^{23}$

Die erste Aussage, die regionale Gültigkeit, lässt sich mittels Quellenuntersuchungen und durch vergleichende Analyse der in Frage kommenden Antiphonen zu bestätigen. Die zweite Behauptung der jüngeren Herkunft würde jedoch der wohl berechtigten Vermutung widersprechen, dass der überzählige Psalmton bis in das Zeitalter noch vor der Systematisierung der acht Töne zurückgeht.

19 Vom Commemoratio brevis (aus der zweiten Hälfte des 9. Jahrhunderts) bis zum Antiphonale monasticum (Solesmes, 1934). Vgl. ERBACHER, op. cit., passim.

20 Conr. ZAB. tract.: „Psalmus peregrinus [In exitu]“; GÜMPEL, Karl-Werner. Die Musiktraktate Conrads von Zabern. Akademie der Wissenschaften und der Literatur. Mainz. Abhandlungen der Geistes- und Sozialwissenschaftl. Kl., I. Jg., Nr. 4. Wiesbaden: Franz Steiner Verlag, 1956, S. 237. - Trad. Holl. II 4, 378: „Et solus psalmus iste In exitu Israel nunc sub ipso [tono] solemniter decantari debet [...]“; BERNHARD, Michael - WITKOWSKA-ZAREMBA, Elżbieta (Hrsg.). TIH, Band II, Die Traktate I - III. Bayerische Akademie der Wissenschaften. Veröffentlichungen der Musikhistorischen Komission, Band 20. München: Verlag der Bayerischen Akademie der Wissenschaften, 2010, S. 280. - FINCK, op. cit., Ppiiiir: „Est adhuc tonus aut tropus, qui uocatur peregrinus, eo quod peregrinus sit, et rarus admodum in cantu, et solummodo in Psalmo, In exitu Israel et caetera. [...]" 21 Über „offizielle” Benennung und Erklärungen des Namens siehe: ERBACHER, op. cit., S. 68-71.

22 HÜSCHEN, Heinrich. Das Cantuagium des Heinrich Eger von Kalkar 1328-1408. Beiträge zur Rheinischen Musikgeschichte, Heft 2. Köln: Staufen-Verlag, 1952, S. 62.

23 NIEMÖLLER, Klaus Wolfgang. Die Musica gregoriana des Nicolaus Wollick. Opus aureum, Köln, 1501, pars I/II. Beiträge zur Rheinischen Musikgeschichte, Heft 11. Köln: Staufen-Verlag, 1955, S. 75. - Vgl. auch: PRASBERG, Balthasar. Clarissima planae atque choralis musicae interpretatio. Basel, 1501. 
Eine keineswegs genügende Anzahl von notierten Beispielen des Tonus peregrinus bieten jedenfalls nur die sporadisch auftauchenden Differenzformel, die Saeculorum amen nach der Antiphon in den Antiphonarien, aber auch in musiktheoretischen Abhandlungen veröffentlichten Psalmdifferenzen und ferner / ausserdem die vor allem in Traktaten notierten Psalmmelodien - entweder über einem Lehrvers oder mit dem ersten Vers des Psalms 113 (siehe Notenbeispiel 1).

a.)

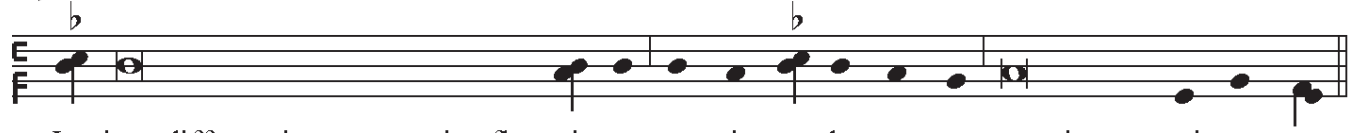

In ista differencia punctus sic fle-cti-tur, et sic e-le-va-tur, et sic ter-mi-na-tur.

b.)

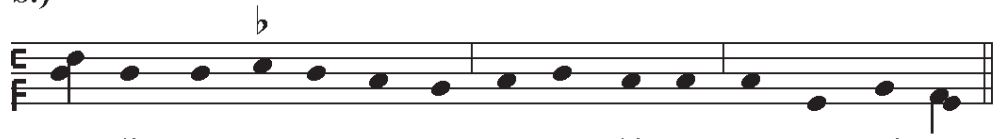

Ta - li te - no-re to-nus can-ta - bi-tur pe - re - gri-nus.

c.)

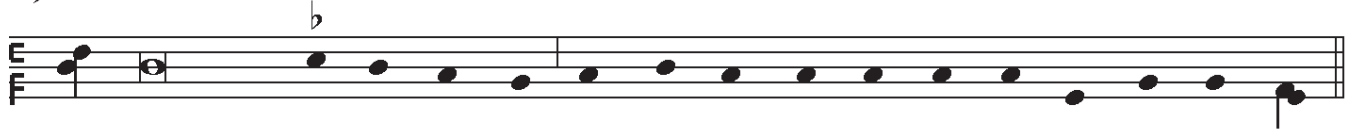

In exitu Israel de E - gy - pto do - mus Ia - cob de po - pu - lo bar - ba - ro.

Notenbeispiel $1^{24}$

Man erforscht die durch Traktaten zur Verfügung stehenden Tonus-peregrinus-Melodien mit dem Ziel, die Variantenbildung der einzelnen Bestandteile des Psalmtons festzustellen. Damit können etliche wesentliche Merkmale im variablen Melodieverlauf in Betracht gezogen werden, die die Grundzüge dieser irregulären Psalmmelodie (auch mit Hilfe von theoretischen Dokumenten) in der lebendigen Gesangspraxis erläutern.

Der erste auffällige Bestandteil des Tonus Peregrinus ist das Initium am Beginn der Psalmformel. Es erscheint in zwei Varianten gemäß den beiden Dialekten des gregorianischen Gesangs. West- und südeuropäische Quellen belegen das von Pes geprägten Initium mit der kleinen Sekunde (s. Notenbeispiel 2a). Diejenige aus Mitteleuropa sind dagegen von einem Terzsprung gekennzeichnet (s. Notenbeispiel 2b). Der Traktat Speculum musicae von Jacobus Leodiensis (Jakob aus Liège) zitiert beide Varianten neben-

24 a/ Ton. Franc. - COUSSEMAKER, Charles-Edmond-Henri de (Hrsg.). Scriptorum de Musica Medii Aevi Nova Series, Vol. II. Paris: A. Durand, 1867, S. 140.; b-c/ Trad. Holl. V 4, 504 und 514 - BERNHARD, Michael - WITKOWSKA-ZAREMBA, Elżbieta (Hrsg.), TIH, Band III, Die Traktate IV - VIII. Bayerische Akademie der Wissenschaften. Veröffentlichungen der Musikhistorischen Komission, Band 21. München: Verlag der Bayerischen Akademie der Wissenschaften, 2011, S. 166. 
einander. ${ }^{25}$ Das zweite, zum mitteleuropäischen germanischen/pentatonischen Dialekt gehörende Initium wurde in der Neuzeit zu einem einzelnen „ $c$ ” reduziert. ${ }^{26}$ Obwohl das Initium einen harmonischen Übergang zwischen der Antiphon und dem Rezitationston bildet, konnte die Psalmformel sogar direkt vom Rezitationston beginnen. Eine Verbindung mit dem 1. Psalmton deutet andererseits ein schrittweise ansteigendes Initium an (Notenbeispiel 2c).

a.)

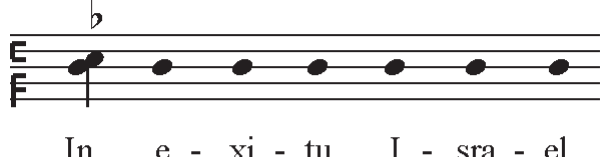

b.)

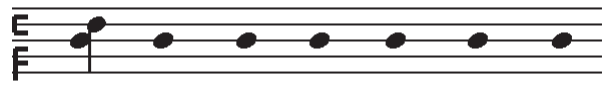

In e - xi - tu I - sra - el ... c.)

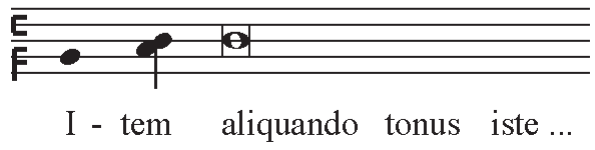

Notenbeispiel $2^{27}$

Als zweiter Bestandteil von maßgebender Bedeutung wird das zweite Initium des Psalmtons wahrgenommen. Einen seltenen jedoch bemerkenswerten Sonderfall stellen manche Beispiele später zentraleuropäischer Quellen dar: nämlich ein Quartinitium unter dem Rezitationston (s. Notenbeispiel 3a). Die allgemein geltende Form des doppelten Initiums überleitet dagegen vom ersten Rezitationston zu dem anderen, und wiederholt gleichzeitig die erste Melodie des Initiums, entweder übereinstimmend oder transponiert (s. Notenbeispiel 3b-c). Das letztere, transponierte Initium erscheint meistens nun leicht verändert: der Pes wurde in zwei separate Töne zerlegt (s. Notenbeispiel 3d). Quellen dieser Variante sind einerseits Traktaten der Hollandrinus-Tradition,

25 BRAGARD, Joseph (ed.). Jacobi Leodiensis Speculum musicae. Corpus Scriptorum de Musica, 3. Rome: American Institute of Musicology, 1968, Vol. 6, S. 251.

26 ERBACHER, op. cit., S. 42-43. Diese Fassung wird auch in Peregrinus-Melodien in der Volkssprache aus dem 20. Jahrhundert erkennbar, und sollte unbezweifelbare Vorgänger in den Gesangbüchern und in der liturgisch-musikalischen Praxis haben. Vgl. SZENDREI, Janka - DOBSZAY, László - RAJECZKY, Benjamin. XVI-XVII. századi dallamaink a népi emlékezetben [Melodien aus dem 16. - 17. Jahrhundert in der mündlichen Tradition des Laienvolkes]. Budapest: Akadémiai Kiadó, 1979, Vol. I, 230-231.

27 a-b/ IAc. Leod. spec. (siehe oben Fn. 25); c/ Guido Dion. 2, 8, 56 - VAN DE KLUNDERT, Sieglinde. Guido von Saint-Denis. Tractatus de tonis. Edition und Studien. Erlangen: Huricane Publishers, 1998, Vol. 2, 132. 
andererseits gedruckte Musiklehrbücher. Im Großen und Ganzen also späte musiktheoretische Werke aus der zentraleuropäischen Region. ${ }^{28}$

a.)

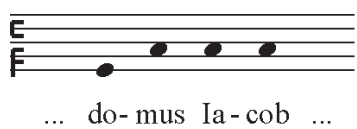

b.)

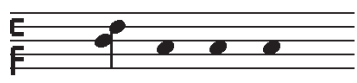

do - mus Ia-cob ...

c.)

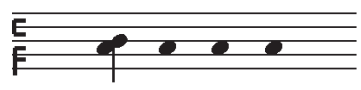

... do-mus Ia-cob...

d.)

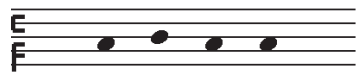

... do-mus Ia-cob ...

Notenbeispiel $3^{29}$

Eine modifizierte Version, die Verschiebung des vorgestellten zweiten Initiums lässt sich schon aus einem etwas früheren Zeitraum, aus dem 14. Jahrhundert nachweisen (s. Notenbeispiel 4a-b). Bei längeren Psalmversen wurden die beiden Arten von doppelten Initien kombiniert eingefügt, damit der zweite Halbvers des Psalmtons einen mehrfach akzentuierten Verlauf erhält (s. Notenbeispiel 4c). Letztendlich wäre aus diesem verschobenen Initium abzuleiten, dass die Rezitation auf dem „G” Tenor scharf abreißt, um dadurch eine Silbe höher herauszuheben. Diese Kadenz (s. Notenbeispiel 4d) - mit den drei vorbereitenden Tönen: „ $a G D$ ” könnte als in Mitteleuropa am häufigsten vorkommende betrachtet werden. Gleichzeitig erscheint dies in den Notierungen der sich auf sechs Töne beschränkenden Kadenzformeln. ${ }^{30}$ Die alternative Variante, die sonst allgemein bekannter ist, enthält den Ton „ $a$ ” gar nicht (s. Notenbeispiel 4e).

28 Z. B. Trad. Holl. II, Trad. Holl. V, Trad. Holl. VII, SZYdlov. - BERNHARD - WITKOWSKA-ZAREMBA (Hrsg.), TIH, Band II, op. cit.; Idem, TIH, Band III, op. cit.; Idem, TIH, Band VI, op. cit. - Siehe auch: LUNDBERG, Mattias. Tonus Peregrinus: The History of a Psalm-tone and its use in Polyphonic Music. Ashgate, $2011, \mathrm{~S}$. $10-11$.

29 a/ LAd. ZAlk. 4, 310. - BERNHARD - WITKOWSKA-ZAREMBA (Hrsg.), TIH, Band VI, op. cit., S. 401; b/ IAc. LeOD. spec. - BRAGARD, op. cit. (s. oben Fn. 25) - c/ Vgl. ERBACHER, op. cit., S. 43. - d/ Siehe Fn. 28.

30 Z. B. Hugo Spechtsh. fol. Liiiir - GÜMPEL, Karl-Werner. Hugo Spechtshart von Reutlingen Flores musicae (1332/42). Akademie der Wissenschaften un der Literatur. Mainz. Abhandlungen der Geistes- und Sozialwissenschaftl. Kl. III. Jg., Nr. 3. Wiesbaden: Franz Steiner Verlag, 1958, S. 167.; IoH. Olom. - SEAY, Albert (Hrsg.). Johannes de Olomons (15th Century). Palma Choralis. Colorado Springs: Colorado College Music Press, 1977, S. 


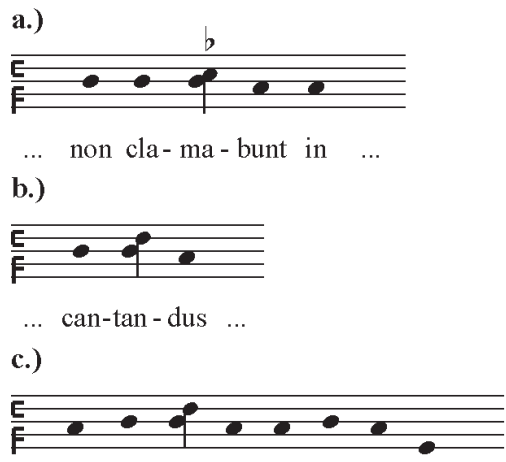

... do-mus Ia-cob de po-pu-lo

d.)

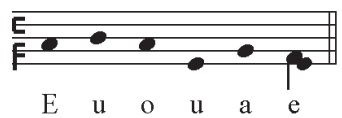

e.)

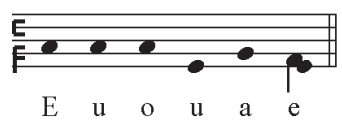

Notenbeispiel $4^{31}$

Einige theoretische Schriften brachten zum Ausdruck, dass sich der Tonus peregrinus nicht ganz in den 8. Modus einfügt und im Rahmen des dargestellten Tonarts fremd wirkt. Aus diesem Grund vervielfachten sich an manchen Orten die Auslegungen der möglichen Moduszuweisungen. Wegen des „D” Finalis des Psalmtons lag eine Zuweisung in den 1. Modus an der Hand. Der aus dem westfränkischen Dialektenkreis stammende Traktat und Tonar aus Saint-Denis teilte diese Melodieformel dagegen als seltene Ausnahme dem 6. Modus zu. Dieselbe Auslegung begegnet man aber auch in einer erstrangigen Quelle der ältesten Hollandrinus-Tradition.

„Est ulterius advertendum quod a regulari intonatione primi toni iam tacta in simplicibus psalmis excipitur intonatio illius psalmi In exitu Israel de Aegypto super antiphonam illam Nos qui vivimus [...]" (IAC. LEOD. spec. Liber 6) ${ }^{32}$

66-67.; KEINSPECK, op. cit.; PRASBERG, op. cit; FELSZTYN, op. cit.; siehe ferner ERBACHER, op. cit., S. 49, Fn. 84. und die Hollandrinus-Traktate: BERNHARD - WITKOWSKA-ZAREMBA (Hrsg.), TIH, passim.

31 a/ Hier. Mor. - Thesaurus Musicarum Latinarum, URL: http://www.chmtl.indiana.edu/tml/13th/ IERTRA1_12GF.gif (abgerufen: 30. 11. 2015); b/ KAISER, Wilfried. Dietrich Tzwyvel und sein Musiktraktat. "Introductorium musicae practicae“ Münster 1513. Marburger Beiträge zur Musikforschung. Heinrich Hüschen (Hrsg.). Marburg, 1968, S. 302.; c/ ANon. Claudifor. - RAUTER, Karl. Der Klagenfurter Musiktraktat von 1430. Tractatus de musica. Klagenfurt: Kärntner, 1989, S. 127, 176.; d/ Siehe Fn. 30.

32 BRAGARD, op. cit., Vol. 6, S. 250. 
„Habet enim irregulare medium intonandi, idest non secundum formam et regulam octavi toni, sed accomodatum potius a sexto tono. Unde et aliqui huiusmodi cantum appellant obliquum, alii peregrinum, alii abortivum." (GuIDo Dion. 2, 8, 60-64) ${ }^{33}$

„[...] vero peregrinum, eo quod in principio formam primi aut 6ti habeat et a communi melodia, que octavo tono subiungitur, discrepat [...]“ (TRAD. Holl. I 2, 15, 10) $)^{34}$

Nicht selten wurde der Tonus peregrinus von gewissen Theoretikern im Zusammenhang mit dem 7. Modus behandelt. Abgeleitet wurde er jedoch nicht aus der Psalmmelodie, sondern aus der zugehörigen Antiphon. Die Gegenüberstellung von mehreren Standpunkten haben auch einige Autoren überlegt, wie z. B. Jerzy Liban aus Polen, der diesbezüglich auch Franchinus Gafurius und Hugo von Spechtshart von Reutlingen namentlich erwähnte. Letztlich stimmte der Anschauung keiner von denen zu, und anstatt der Modi des Tetrardus den 1. Ton wählte.

„Franchinus, qui aetate nostra, inter Musicos facile principes extitit: Peregrinum septimo tono (cum Ambrosianis) suis adscripsit. Hugo sacerdos Reutlingensis, natione Germanus, qui ante duo saecula, ... de musica et sensit et scripsit, octauo tono, peregrinum applicat: Nam Ambrosiani eius toni antiphonam, scilicet Nos qui viuimus, in G graui incipiunt: [...] Gregoriani in C graui. Quare, aliorum pace, hunc tonum magis referre primum et primam eius differentiam, quam aut septimum aut octauum dixerim. Qui habet aures audiendi, audiat." (Liban: De musicae laudibus oratio, Fiiir-v)

Allerdings spielte die Unregelmäßigkeit des Psalmtons in der Modalitätsbestimmung des Tonus peregrinus offensichtlich eine geringere Rolle. ${ }^{35}$ Viel schwerwiegender fiel die Verknüpfung des Tons mit der darauffolgenden Antiphonenmelodie, bzw. die Einordnung einer kleinen Gruppe von Antiphonen in das System der acht Modi.

Von den ältesten Dokumenten ${ }^{36}$ bis zu den späteren Traktaten wurden vor allem Nos qui vivimus, und manche mit ihr verwandten Antiphonen als Beispiele für Tonus peregrinus zitiert (s. Notenbeispiel 5a). Der Psalmton des Peregrinus stand im Einklang mit dem Gestalt der plagalen G-Melodie der Antiphon: sie nahm die untere Lage des Ambitus ein - wie dies auch durch die Theoretiker festgestellt wurde. ${ }^{37}$ In den frühen süddeutschen

33 VAN DE KLUNDERT, op. cit., Vol. 2, S. 132.

34 BERNHARD - WITKOWSKA-ZAREMBA (Hrsg.), TIH, Band II, op. cit., S. 129.

35 Siehe auch ERBACHER, op. cit., S. 6-7.

36 Cомм. Br. Vgl. SCHMID, Hans. Musica et Scholica Enchiriadis una cum aliquibus tractatulis adiunctis. Bayerische Akademie der Wissenschaften. Veröffentlichungen der Musikhistorischen Kommission, Band 3. München: verlag der Bayerischen Akademie der Wissenschaften, 1981, S. 165. - Vgl. die moderne Übertragung in BAILEY, Terence (Hrsg.). Commemoratio brevis de tonis et psalmis modulandis. Ottawa: The University of Ottawa Press, 1979, S. 55-57.

37 Mod. Autenticus (Pseudo-Hucbald: De modis): „Parapter vero quartus contingit totum octavum, sed non ascendit in alteram vocem, et mediocris in ipsam finitur..." Zitat bei ATKINSON 1982, op. cit., S. 44. 
Tonaren wurde die Antiphon Nos qui vivimus dem 8. Ton aufgrund seiner zusätzlichen Kadenz zugeordnet, und ausnahmsweise auch dem 7. Ton. ${ }^{38}$

Was für ein Bild zeichnet sich mithilfe der Offiziumsgesangbücher? Die Zuweisung von einer Psalmkadenz der Art Tonus peregrinus war für Choralhandschriften der süddeutschen Benediktiner charakteristisch; ${ }^{39}$ von den religiösen Orden fand dieselbe Praxis auch bei den Franziskanern und den ungarischen Paulinern Anwendung. ${ }^{40}$ Von den Diözesen war es in Passau, ${ }^{41}$ Augsburg (s. Notenbeispiel 5b) ${ }^{42}$ und Breslau (Wrocław) ${ }^{43}$ eingebürgert. In Ungarn wurde die Antiphon mit dem 8. Ton verbunden (s. Notenbeispiel 5c). ${ }^{44}$

Es scheint unmöglich, die divergierende Moduswahl auf einer vollständigen geographischen Landkarte wiederzugeben. In zahlreichen kirchlichen Zentren (Klosterneuburg, Prag, Krakau) war nämlich als die im Sonntagsvesper bevorzugte Antiphon Domus Jacob. Eine typische Variante der Antiphon entstand durch Transposition (s. Notenbeispiel 5d); der

a.)

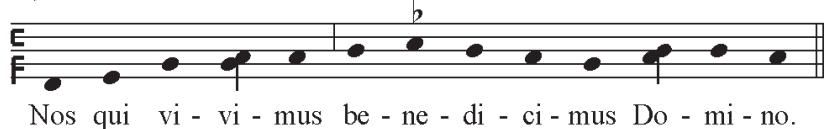

b.)

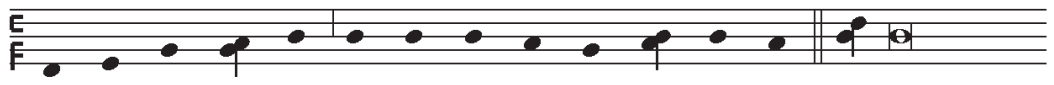

Nos qui vi - vi - mus be - ne - di - ci-mus Do - mi - no. In exitu Israel...

c.)

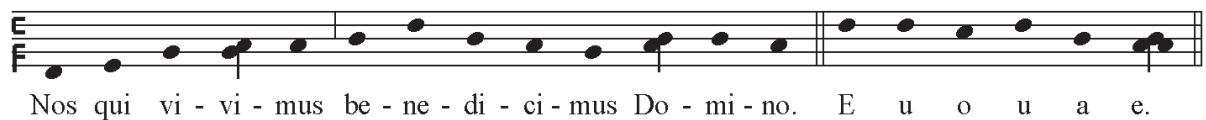

d.)

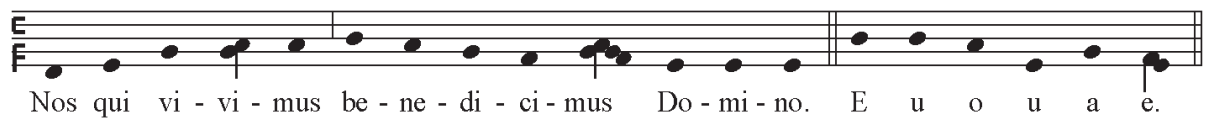

Notenbeispiel $5^{45}$

38 Vgl. RAUSCH 1999, op. cit., S. 177. (Tabelle „Tonare im Vergleich”)

39 Siehe z. B. CH-SGs 388; D-Mbs Clm 4303; F-Pn lat. 12044 - Die einzelnen Quellenangaben stammen aus dem CANTUS-Datenbank. Siehe im Internet: http://cantusdatabase.org/node/377377 (Antiphon Nos qui vivimus); http://cantusdatabase.org/sources (Quellenliste). Abgerufen: 01. 12. 2015.

40 Siehe z. B. CH-Fco 2; DOBSZAY, László - SZENDREI, Janka (Hrsg.). Monumenta Monodica Medii Aevi, Band V, Antiphonen. 3 Bde. Kassel etc.: Bärenreiter, 1999, No. 8468 (im Teilband 3).

41 Antiphonale Pataviense (Wien, 1519) [Faksimile]. SCHLAGER, Karlheinz (Hrsg.). Das Erbe deutscher Musik, Vol. 88. Kassel etc.: Bärenreiter, 1985, f. 28v; CZ-Pu, I D 20, f. 33r.

42 DK-Kk $34498^{\circ}$ IV, f. 63v; DK-Kk $34498^{\circ} \mathrm{XV}$, f. 15v.

43 PL-WRu R 503, f. 37v; PL-WRk Rps. 168, p. 120.

44 DOBSZAY - SZENDREI (Hrsg.), op. cit., No. 8468.

45 a/ Comm. BR. (s. Fn.36); b/ s. Fn. 42; c/ Antiphonale Strigoniense - SK-BRm EC. Lad. 6. (s. DOBSZAY SZENDREI (Hrsg.), op. cit., No. 8468.; d/ F-Pn lat. 15181, f. 184v-185r. 
Kadenzwechsel von „G” zu „D” begründete einen Übergang zum Tonus peregrinus, das aus Pariser Gesangbücher, und ferner aus Zisterzienser - und Karmelitenkloster bekannt ist. ${ }^{46}$

Der „D” Finalis brachte den Rezitationston „F” mit sich, und gleichzeitig eine Eingliederung in den 2. Modus. (Notenbeispiel 6a) Der ebenso de psalterio gesungene Antiphon In ecclesiis bietet uns genügend Stoff für eine weitere Untersuchung: die mittelalterliche Theorie ließ dieses Stück als tonal doppelgesichtig bis zum 14. Jahrhundert fortleben. ${ }^{47}$ Eine Verbindung dieser Antiphon mit dem Tonus peregrinus wird jedoch keineswegs durch unsere Erfahrungen bei praktischen Offiziumsgesangbücher unterstützt. Varianten im 2. Ton können nur vereinzelt belegt werden ${ }^{48}$ Die sonst überwiegend einheitlich im 8. Ton gedeutete Antiphonenmelodie weist inzwischen nichts mehr als mannigfaltige Incipitvarianten auf (s. Notenbeispiele 6b-e).

a.)

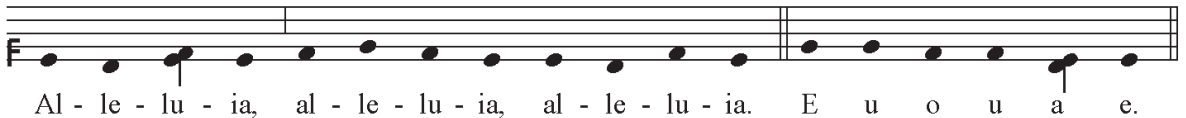

b.)

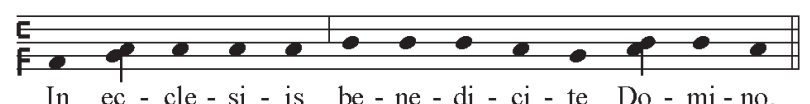

c.)

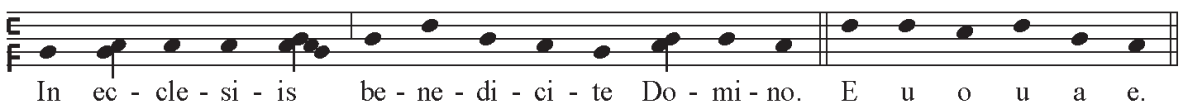

d.)

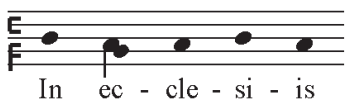

e.)

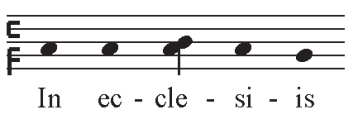

Notenbeispiel $6^{49}$

Die tonalen Varianten der Antiphonenfamilie Martyres/Angeli/Virgines Domini lieferten schon früher eine Grundlage für vielfältigen Beobachtungen ${ }^{50}$ Bemerkenswert ist jedoch,

46 Vgl. F-Pn lat. 15181; F-Pn n. a. lat. 1411; CDN-Hsmu M2149.L4; A-Wn 1799**; D-MZb A; PL-Kkar 4 (Rkp 20). Die einzelnen Quellenangaben stammen aus dem CANTUS-Datenbank (siehe oben Fn. 39).

47 Wohlbekannt ist als letzter Beispiel des vierten „Parapters” (vgl. oben Fn. 2), wird aber im späteren theoretischen Literatur ausschliesslich in IAC. LEOD. spec. zitiert.

48 Vgl. CLAIRE 1975, op. cit., S. 91.

49 a/ Ibid.; b/ A-Gu 29, f. 74r; c/ DOBSZAY - SZENDREI (Hrsg.), op. cit., No. 8014 (im Teilband 3); d/ PL-WRu R 503, 40r; e/ PL-Kk Ms. 47, f. 115r.

50 LIPPHARDT, Walther. Der karolingische Tonar von Metz. Liturgiewissenschaftliche Quellen und Forschun- 
dass während der Jahrhunderte die Melodieformel von Tonus peregrinus verhältnismäßig selten zu den vorher genannten Antiphonen angeknüpft wurde. ${ }^{51}$ Im Gegenteil zu den „klassischen” Moduswechsel: statt des plagalen Tetrardus (8. Modus) wurde der plagale Deuterus (4. Modus) bestimmt. Darüber besitzt man genügende Aussagen seit den frühen sanktgallischen Tonaren. ${ }^{52}$ Unserer Beobachtungen nach sollten den als voneinander abweichend fassbaren Tonarten von G- und E-Modus auch unterschiedliche Antiphonenincipits zugehören. (Notenbeispiel 7) Das tetratonische Incipit erscheint zweifach transponiert: entweder auf „D” oder - um eine Sekunde höher - auf „E”. Diese letztere, höhere Version entspricht völlig dem typischen Eröffnungsabschnitt der Antiphonenmelodie im 4. Ton. Aus der Analyse mehrerer Beispiele wird aber sichtbar, dass eine Abrundung im irgendeinen Modus unabhängig vom Verlauf der Antiphon eintritt. So sind Varianten nicht nur im 4., sondern auch im 1. Modus entstanden (s. Notenbeispiel 7c)

a.)

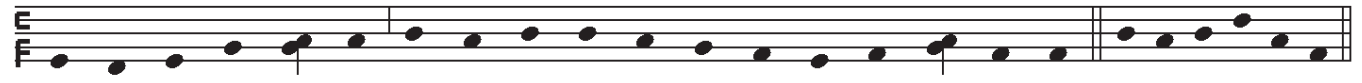

Mar-ty-res Do-mi - ni Do-mi-num be-ne-di-ci-te in ae-ter-num. E $u$ o $u$ a e.

b.)

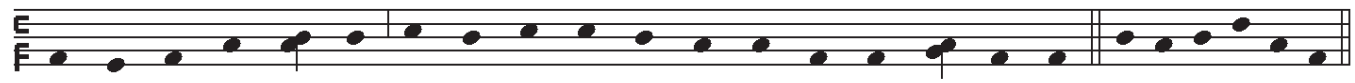

An-ge- li Do-mi - ni Do-mi-num be-ne-di-ci-te in ae-ter-num. E u o u a e.

c.)

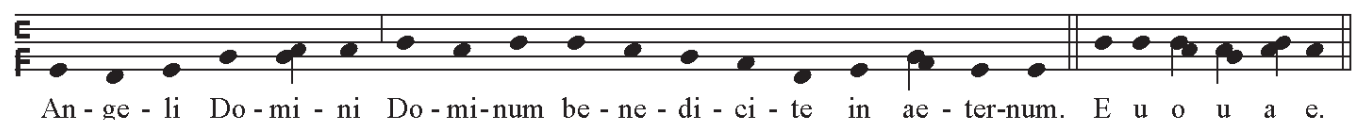

An-ge - li Do-mi - ni Do-mi-num be-ne-di-ci-te in ae-ter-num. E $u$ o $u$ a e.

Notenbeispiel $7^{53}$

Die verschiedenen melodischen und tonalen Gestalte der Antiphonen waren im Mittelalter zusammenfassend unter den Regionen und kirchlichen Institutionen, wie folgt, verteilt:

gen, 43. Münster: Aschendorffsche Verlagsbuchhandlung, 1965, S. 288; ERBACHER, op. cit., S. 25; STEINER, Ruth. Antiphons for Benedicite at Lauds. Journal of the Plainsong and Mediaeval Music Society 5, 1984, S. 1-17. (hier: 11-12, Fn. 26); DOBSZAY, László. Corpus antiphonarum. Európai örökség és hazai alakitás [Europäisches Erbe und heimische Gestaltung]. Budapest: Balassi Kiadó, 2003, S. 236-237.

51 Nach Stichproben werden folgende Handschriften vermerkt, welche zu diesen Antiphonen den Peregrinus-Ton zufügen: CH-E 611, f. 257r; D-BAs lit. 25, f. 115v; CZ-HKm II A 3, f. 179v; CZ-Pn XV A10, f. 346v; PL-WRu R 503, f. 199r, 223v.

52 OMLIN, Ephrem. Die sanktgallischen Tonarbuchstaben. Veröffentlichungen der Gregor. Akademie zu Freiburg (Schweiz). Fellerer, K. G. (Hrsg.), Heft 18. Regensburg: Verlag Friedrich Pustet, 1934, S. 313. - In der Hollandrinus-Tradition ist ein transponierter 4. Modus in SzydLov. nachweisbar. S. BERNHARD - WITKOWSKA-ZAREMBA, TIH, Band VI, op. cit., S. 525.

53 a/ D-Mbs; Clm 4304, f. 30r; b/ D-Mbs Clm 4305, f. 185r; c/ TR-Itks 42 - SZENDREI, Janka (Hrsg.). The Istanbul Antiphonal about 1360. Musicalia Danubiana, Vol. 18. Budapest: Akadémiai Kiadó, 2002, f. 238r. 
Die Fassung im 4. Ton ist zumeist in monastischen Kodizes aufzufinden, ${ }^{54}$ die Fassung im 8. Ton enthalten Handschriften aus süddeutschem und zentraleuropäischem Gebiet, die größtenteils dem weltlichen Usus zugehörig sind ${ }^{55}$ Für die Praxis der Franziskaner ist der Wechsel zwischen den beiden tonalen Varianten kennzeichnend.$^{56}$ Zur Melodiefassung im 1. Ton gibt es keine verfügbaren theoretischen Quellen. Die Überlieferung dieser Variante stammt aus zentralen ungarischen Offiziumshandschriften ${ }^{57}$ und nebenher aus Kodizes der Zisterzienser und der Karmelitenkloster aus verschiedensten Regionen Europas. ${ }^{58}$
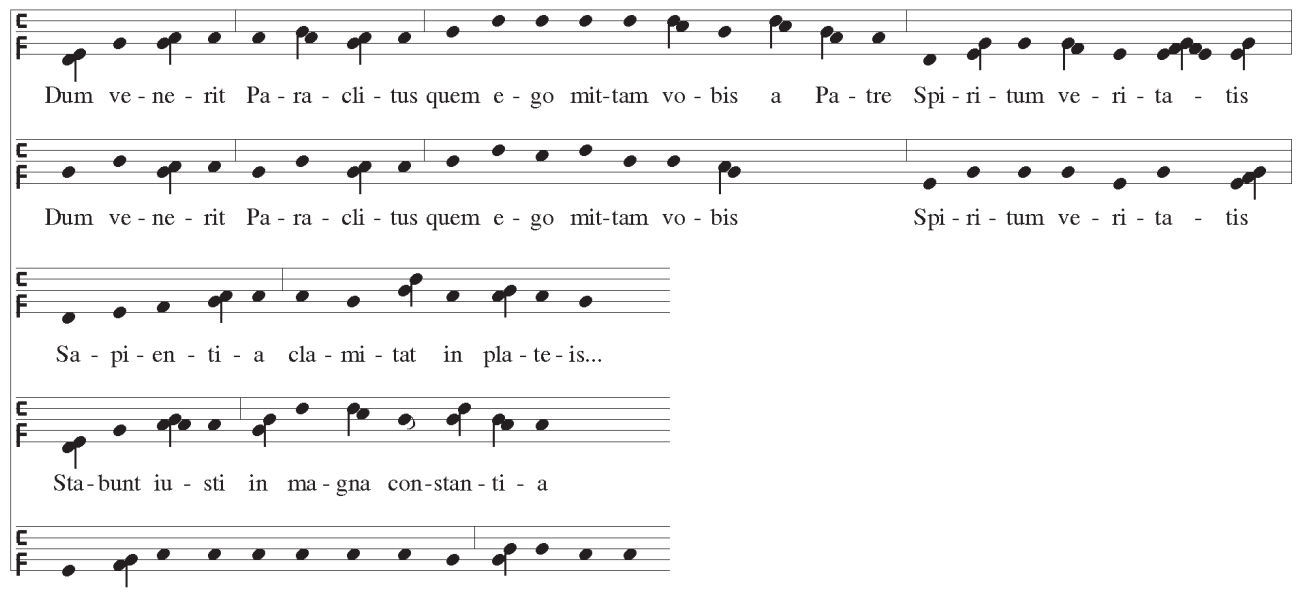

Di - xit Do-mi-nus mu - li - e - ri Ca-na-ne-ae

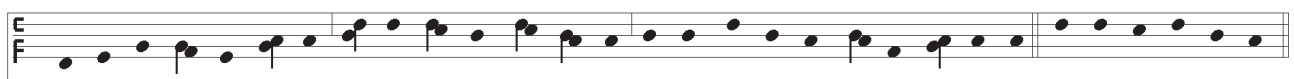

qui a Pa-tre pro-ce-dit il- le te-sti-mo-ni-um per-hi-be-bit de me al-le-lu-ja. E u o u a e

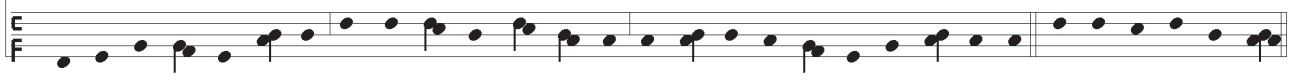

qui a Pa-tre pro-ce-dit il- le te-sti-mo-ni-um per-hi-be-bit de me al-le-lu-ja. E u o u a e

Notenbeispiel $8^{59}$

54 Vgl. Fn. 53/a-b/; und auch A-Gu 29 bzw. 30; I-Lc 601. CANTUS-Datenbank: http://cantusdatabase.org/ id/001399 und http://cantusdatabase.org/id/003717 (abgerufen: 01. 12. 2015)

55 Klosterneuburg, Passau, Salzburg, Augsburg. Vgl. A-KN 1012, 1018; A-VOR 287; DK-Kk $34498^{\circ}$ XII und XVI im CANTUS-Datenbank. Ferner: CZ-Pn I D 20, f. 196r. - Als Ausnahme können Kodizes aus Benediktinerklöster in Mähren und Tschechien erwähnt werden: CZ-R R 17, f. 209v, 262v; CZ-Pu XIII C 4, f. 10r, 55r.

56 DOBSZAY - SZENDREI (Hrsg.), op. cit., Nr. 1383, 1384 (im Teilband 1); ferner Ton. Franc. - COUSSEMAKER, op. cit., S. 128; CH-Fco 2 im CANTUS-Datenbank.

57 DOBSZAY - SZENDREI (Hrsg.), ibid.

58 Vgl. F-Pn n. a. lat. 1412; CDN-Hsmu M2149.L4; A-Wn 1799**; D-MZb A; PL-Kkar 4 (Rkp 20) im CANTUS-Datenbank (s. Fn.39 und 54.)

59 Dum venerit paraclitus: CH-E 611, f. 112r; TR-Itks 42, f. 109r. Stabunt iusti: TR-Itks, f. 175r. Die übrigen Beispiele alle aus DOBSZAY - SZENDREI (Hrsg.), op. cit. (siehe Tab. 1). 
Das theoretische Schrifttum präsentiert im Zusammenhang mit dem Tonus peregrinus noch einige weiteren Antiphonenincipits. (Eine Liste von ihnen siehe in der Tab. 1) Die Offiziumsgesangbücher, die die Gesangspraxis widerspiegeln, behandelten die fraglichen Antiphonen im großen Ganzen nicht der Äußerungen der Theoretiker entsprechend. Letztere hatten einfach Incipits von Antiphonen im 8. Modus zum Tonus peregrinus zugeordnet: sogar Incipits einiger Stücke von ziemlich erweiterter Form wie Dixit Dominus mulieri, Cum venerit Paraclitus und Sapientia clamitat. (S. Notenbeispiel 8) Der einzige Grund für die Zuweisung dieser Antiphonen der „peregrina” Psalmkadenz war die Analogie der Incipitsmelodie zu jener der Nos qui vivimus. Im Falle der Apud Dominum, einer Antiphon im 4. Ton handelt es sich wiederum um das Transponieren desselben Incipits (wie dies schon bei der Antiphonengruppe Martyres oben geschildert wurde).

Es ist sicherlich eine Klassifikation nach formalen Entsprechungen, ein mechanisches Verfahren, was man in den späteren Lehrbüchern beobachten kann. Man begegnet eine Betrachtungsweise, die gar nicht der praktisch orientierten Choralüberlieferung von Mitteleuropa entspricht. Die Gegenüberstellung von spätmittelalterlichen musiktheoretischen Kompendien und Choralhandschriften hat uns gezeigt, dass in den Abhandlungen der Tonus peregrinus bloß zu einem musiktheoretischen Phänomen geworden ist, es diente als ein gebräuchliches Requisit, und letztendlich brauchte man dazu gar keine wahren, lebendigen Beispiele, sondern musste lediglich als angewandtes Illustrationsmaterial herhalten.

\begin{tabular}{|c|c|c|c|c|}
\hline & Theor. Quellen & $\begin{array}{l}\text { Psalmkadenz in } \\
\text { Gesangbüchern }\end{array}$ & Charakteristika & $\begin{array}{l}\text { DOBSZAY - } \\
\text { SZENDREI (Hrsg.) }\end{array}$ \\
\hline $\begin{array}{l}\text { Cum/Dum vene- } \\
\text { rit Paraclytus ... } \\
\text { vobis Spiritum }\end{array}$ & $\begin{array}{l}\text { Ton. Franc. }{ }^{60} \\
\text { Sarum ton. }{ }^{61} \\
\text { QUAT. PRINC. }^{62} \\
\text { WALT. OdInGT. }{ }^{63} \\
\text { IOH. GARL. plan. }{ }^{64} \\
\text { IAC. LEOD. spec. }{ }^{65}\end{array}$ & $\begin{array}{l}\text { 8. Modus } \\
\text { Diff. g1/g2 }\end{array}$ & $\begin{array}{l}\text { zweifache (va- } \\
\text { riable) Incipits }\end{array}$ & Nr. 8400 \\
\hline
\end{tabular}

60 COUSSEMAKER, op. cit., S. 140.

61 FRERE, Walter Howard. Antiphonale Sarisburiense, Vol I. Introduction and Indices. Reprint. Farnborough: Gregg Press, 1966, S. 85, 93.; Idem. The Use of Sarum, Vol. II. The Ordinal and Tonal. Cambridge: University Press, 1901, S. ixiij.

62 COUSSEMAKER, Charles-Edmond-Henri de (Hrsg.). Scriptorum de Musica Medii Aevi Nova Series. Vol. IV. Paris: A. Durand, 1876, S. 245.

63 COUSSEMAKER, Charles-Edmond-Henri de (Hrsg.). Scriptorum de Musica Medii Aevi Nova Series. Vol. I. Paris: A. Durand, 1864, S. 291.

64 Ibid., S. 174.

65 BRAGARD, op. cit., Vol. 6, S. 306. 


\begin{tabular}{|c|c|c|c|c|}
\hline & Theor. Quellen & $\begin{array}{l}\text { Psalmkadenz in } \\
\text { Gesangbüchern }\end{array}$ & Charakteristika & $\begin{array}{l}\text { DOBSZAY - } \\
\text { SZENDREI (Hrsg.) }\end{array}$ \\
\hline $\begin{array}{l}\text { Sapientia cla- } \\
\text { mitat }\end{array}$ & $\begin{array}{l}\text { Ton. Franc. } \\
\text { Sarum ton. } \\
\text { TRAD. Holl. II }{ }^{66} \\
\text { TrAD. Holl. VII }{ }^{67} \\
\text { ANON. Claudi- } \\
\text { for. }^{68} \\
\text { Ton. Vratisl. }^{69}\end{array}$ & $\begin{array}{l}\text { 8. Modus } \\
\text { Diff. g1/g2 }\end{array}$ & $\begin{array}{l}\text { incipit nach } \\
\text { dem Muster von } \\
\text { Nos qui vivimus }\end{array}$ & Nr. 8380 \\
\hline Stabunt iusti & AnON. Claudifor. & $\begin{array}{l}\text { 8. Modus } \\
\text { Diff. g1/g2 }\end{array}$ & & - \\
\hline $\begin{array}{l}\text { Dixit Dominus } \\
\text { mulieri }\end{array}$ & $\begin{array}{l}\text { TRAD. Holl. XXI- } \\
\text { II }^{71}\end{array}$ & $\begin{array}{l}\text { 8. Modus } \\
\text { Diff. g1/g2/c2 }\end{array}$ & & Nr. 8486 \\
\hline Apud Dominum & $\begin{array}{l}\text { Trad. Holl. XXI }{ }^{72} \\
\text { Trad. Holl. XXIII }\end{array}$ & 4. Modus & $\begin{array}{l}\text { transponiertes } \\
\text { Incipit }\end{array}$ & Nr. 4008 \\
\hline
\end{tabular}

Tab. 1. Antiphonen in loser Verbindung mit Tonus peregrinus.

\section{Bibliography ${ }^{73}$}

\section{Manuscripts cited}

Antiphonarium OSB, St. Lambrecht, saec. 14 (2 vols.)

A-Gu 29 bzw. 30

http: / $/$ www.literature.at/ viewer.alo?objid $=1138 \&$ page $=150$ \&viewmode $=$ overview

Antiphonarium OSB, Einsiedeln, saec. 14

CH-E 611

http://www.e-codices.unifr.ch/en/sbe/0611/bindingA/0/Sequence-1025

Antiphonarium OFM, Fribourg, saec. 13/14

CH-Fco 2

http://www.e-codices.unifr.ch/en/fcc/0002/50v

Antiphonarium, Hradec Králové, saec. 15

Breviarium notatum Pragense, saec. 14/2

CZ-HKm II A 3

CZ-Pn XV A10

http://www.manuscriptorium.com/apps/index.php?direct=record\&pid=AIPDIG-NMP XV_A_10 0MGV3O0-cs

Antiphonarium Pataviense, saec. 14

CZ-Pu I D 20

Antiphonarium OSB in monaterio S. Georgii in castro Pragensi, saec. 13/14

CZ-Pu XIII C 4

66 BERNHARD - WITKOWSKA-ZAREMBA, TIH, Band II, S. 278.

67 BERNHARD - WITKOWSKA-ZAREMBA, TIH, Band III, S.

68 RAUTER, op. cit., S. 127, 176.

69 BERNHARD - WITKOWSKA-ZAREMBA, TIH, Band VI, S. 704.

70 Eine Ausnahme bildet die Tonus peregrinus Differenz im Kodex CH-E 611 (f. 128v). Sie wurde aber in Stelle einer weggekratzten früheren Differenz im 8. Modus notiert.

71 BERNHARD - WITKOWSKA-ZAREMBA, TIH, Band VI, op. cit., 101.

72 BERNHARD - WITKOWSKA-ZAREMBA, TIH, Band $V$, S.

73 All internet downloads cited in the following bibliography were carried out at latest 07. 12. 2015. 
http://www.manuscriptorium.com/apps/index.php?direct=record\&pid=AIPDIG-NKCR_ XIII_C_4 1GXDV7F-cs

Antiphonarium OSB monasterii Rajhrad, 1313

CZ-R R 17

http://www.manuscriptorium.com/apps/index.php?direct=record\&pid=AIPDIGBOPPRBR_17 0DDIM00-cs

Antiphonarium Bambergense, saec. 13

D-BAs lit. 25

Antiphonarium OSB S. Ulrich et Afra, Augsburg, 1519

D-Mbs Clm 4304

http://www.uni-regensburg.de/Fakultaeten/phil_Fak_I/Musikwissenschaft/cantus/microfilm/clm4304/index.html

Antiphonarium OSB S. Ulrich et Afra, Augsburg, 1459

D-Mbs Clm 4305

http://www.uni-regensburg.de/Fakultaeten/phil_Fak_I/Musikwissenschaft/cantus/microfilm/clm4305/index.html

Antiphonarium Augustense, pars IV, 1580c.

DK-Kk $34498^{\circ}$ IV

http://www.uni-regensburg.de/Fakultaeten/phil_Fak_I/Musikwissenschaft/cantus/microfilm/copenhagen/vol4/Antiphonarium Augustense, pars XII, 1580c. DK-Kk $34498^{\circ}$ XII

http://www.uni-regensburg.de/Fakultaeten/phil_Fak_I/Musikwissenschaft/cantus/microfilm/copenhagen/vol12/

Antiphonarium Augustense, pars XV, 1580c.

DK-Kk $34498^{\circ}$ XV

http://www.uni-regensburg.de/Fakultaeten/phil_Fak_I/Musikwissenschaft/cantus/microfilm/copenhagen/vol15/

Antiphonarium Augustense, pars XVI, 1580c.

DK-Kk $34498^{\circ}$ XVI

http://www.uni-regensburg.de/Fakultaeten/phil_Fak_I/Musikwissenschaft/cantus/microfilm/copenhagen/vol16/

Breviarium notatum, Paris, Notre-Dame, 1300c.

F-Pn lat. 15181

http:/ /gallica.bnf.fr/ark:/12148/btv1b8447768b/f1124.item

Antiphonarium Cracoviense, 1457

Antiphonarium Vratislaviense, saec. 14

PL-Kk Ms. 47

Antiphonarium Vratislaviense, saec. 15

PL-WRu R 503

Antiphonale Strigoniense, saec. 15/2 -

PL-WRk Rps. 168

http://cantus.sk/source/14018

SK-BRm EC. Lad. 6.

\section{Printed source material}

COCHLAEUS, Johannes. Tetrachordum musices. Nürnberg, 1514. http://www.chmtl.indiana.edu/ tml/16th/COCTET3_TEXT.html

FELSZTYN, Sebastian. Opusculum musicae compilatum. Krakau, 1517. Reprint: Monumenta Musicae in Polonia. Series D. Bibliotheca Antiqua IX. MORAWSKI, Jerzy (Hrsg.). Kraków: Polskie Wydawnictwo Muzyczne, 1978.

FINCK, Hermann. Practica musica. Wittemberg, 1556. http://www.chmtl.indiana.edu/tml/16th/ FINPRA_TEXT.html

KEINSPECK, Michael. Lilium musicae planae. Augsburg, 1500. http://bildsuche.digitale-sammlungen.de $/$ index.html?c=viewer\&l=en\&bandnummer $=$ bsb00033872\&pimage $=00029 \& v=150 \&$ nav

LIBAN, Jerzy. De musicae laudibus oratio. Krakau, 1540. Reprint: Monumenta Musicae in Polonia, Series D. Bibliotheca Antiqua VIII. MORAWSKI, Jerzy (Hrsg.). Kraków: Polskie Wydawnictwo Muzyczne, 1975. 
ORNITOPARCHUS, Andreas. Musice Active Micrologus. Leipzig, 1517. Reprint: A Compendium of Musical Practice. Musice active micrologus by Andreas Ornithoparchus [...] REESE, Gustave (ed.). New York: Dover, 1973.

PRASBERG, Balthasar. Clarissima planae atque choralis musicae interpretatio. Basel, 1501. Edition: BOHN, P. (Hrsg.). in Caecilia. Organ für katholische Kirchenmusik, Jg. 15-16. Trier, 1876-1877.

TZWYVEL, Dietrich. Introductorium musicae practicae. Münster, 1513. Edition: KAISER, Wilfried. Dietrich Tzwyvel und sein Musiktraktat. „Introductorium musicae practicae“ Münster 1513. Marburger Beiträge zur Musikforschung. Heinrich Hüschen (Hrsg.). Marburg, 1968.

WOLLICK, Nicolaus. Opus aureum. Köln, 1501. Edition: NIEMÖLLER, Klaus Wolfgang. Die Musica gregoriana des Nicolaus Wollick. Opus aureum, Köln, 1501, pars I/II. Beiträge zur Rheinischen Musikgeschichte, Heft 11. Köln: Staufen-Verlag, 1955.

\section{Editions}

BAILEY, Terence (Hrsg.). Commemoratio brevis de tonis et psalmis modulandis. Ottawa: The University of Ottawa Press, 1979.

BECK, Carl (Hrsg.). Flores musice omnis cantus gregoriani von Hugo von Reutlingen. Stuttgart: Literarischer Verein, 1868.

BERNHARD, Michael - WITKOWSKA-ZAREMBA, Elżbieta (Hrsg.). Traditio Iohannis Hollandrini, Band II, Die Traktate I - III. Bayerische Akademie der Wissenschaften. Veröffentlichungen der Musikhistorischen Komission, Band 20. München: Verlag der Bayerischen Akademie der Wissenschaften, 2010.

BERNHARD, Michael - WITKOWSKA-ZAREMBA, Elżbieta (Hrsg.), Traditio Iohannis Hollandrini, Band III, Die Traktate IV - VIII. Bayerische Akademie der Wissenschaften. Veröffentlichungen der Musikhistorischen Komission, Band 21. München: Verlag der Bayerischen Akademie der Wissenschaften, 2011.

BERNHARD, Michael - WITKOWSKA-ZAREMBA, Elżbieta (Hrsg.). Traditio Iohannis Hollandrini, Band V, Die Traktate XV - XXI. Bayerische Akademie der Wissenschaften. Veröffentlichungen der Musikhistorischen Komission, Band 23. München: Verlag der Bayerischen Akademie der Wissenschaften, 2014.

BERNHARD, Michael - WITKOWSKA-ZAREMBA, Elżbieta (Hrsg.), Traditio Iohannis Hollandrini, Band VI, Die Traktate XXII - XXVI, Ladislaus de Zalka und Szydlovita, Appendices [...]Bayerische Akademie der Wissenschaften. Veröffentlichungen der Musikhistorischen Komission, Band 24. München: Verlag der Bayerischen Akademie der Wissenschaften, 2015.

BRAGARD, Joseph (ed.). Jacobi Leodiensis Speculum musicae. Corpus Scriptorum de Musica, 3. Rome: American Institute of Musicology, 1968, Vol. 6.

COUSSEMAKER, Charles-Edmond-Henri de (Hrsg.). Scriptorum de Musica Medii Aevi Nova Series. Vol. I. Paris: A. Durand, 1864.

COUSSEMAKER, Charles-Edmond-Henri de (Hrsg.). Scriptorum de Musica Medii Aevi Nova Series, Vol. II. Paris: A. Durand, 1867.

COUSSEMAKER, Charles-Edmond-Henri de (Hrsg.). Scriptorum de Musica Medii Aevi Nova Series. Vol. IV. Paris: A. Durand, 1876.

GÜMPEL, Karl-Werner. Die Musiktraktate Conrads von Zabern. Akademie der Wissenschaften und der Literatur. Mainz. Abhandlungen der Geistes- und Sozialwissenschaftl. Kl., I. Jg., Nr. 4. Wiesbaden: Franz Steiner Verlag, 1956. 
GÜMPEL, Karl-Werner. Hugo Spechtshart von Reutlingen Flores musicae (1332/42). Akademie der Wissenschaften un der Literatur. Mainz. Abhandlungen der Geistes- und Sozialwissenschaftl. Kl. III. Jg., Nr. 3. Wiesbaden: Franz Steiner Verlag, 1958.

HÜSCHEN, Heinrich. Das Cantuagium des Heinrich Eger von Kalkar 1328-1408. Beiträge zur Rheinischen Musikgeschichte, Heft 2. Köln: Staufen-Verlag, 1952.

KAISER, Wilfried. Dietrich Tzwyvel und sein Musiktraktat. „Introductorium musicae practicae “ Münster 1513. Marburger Beiträge zur Musikforschung. Heinrich Hüschen (Hrsg.). Marburg, 1968.

MATHIAS, F. X. (Hrsg.). Der Straszburger Chronist Königshofen als Choralist. Sein Tonarius. Graz: Styria, 1903.

MÜLLER, Hermann. Der tractatus musicae scientiae des Gobelinus Person. Kirchenmusikalisches Jahrbuch, Jg. 20, 1907, S. 177-196.

RAUSCH, Alexander (Hrsg.). Opusculum de musica ex traditione Iohannis Hollandrini. A Commentary , Critical Edition and Translation. Ottawa: The Institute of Meidaeval Music, 1997.

RAUSCH, Alexander. Die Musiktraktate des Abtes Bern von Reichenau. Musica Mediaevalis Europae Occidentalis, Bd. 5. Walter Pass (Hrsg.). Tutzing: Schneider, 1999.

RAUTER, Karl. Der Klagenfurter Musiktraktat von 1430. Tractatus de musica. Klagenfurt: Kärntner, 1989.

SCHLAGER, Karlheinz (Hrsg.). Antiphonale Pataviense (Wien, 1519) [Faksimile]. Das Erbe deutscher Musik, Vol. 88. Kassel etc.: Bärenreiter, 1985.

SCHMID, Hans. Musica et Scholica Enchiriadis una cum aliquibus tractatulis adiunctis. Bayerische Akademie der Wissenschaften. Veröffentlichungen der Musikhistorischen Kommission, Band 3. München: verlag der Bayerischen Akademie der Wissenschaften, 1981.

SMITS VAN WASBERGHE, Joseph (Hrsg.). Iohannis Affligemensis: De Musica cum Tonario. Corpus Scriptorum de Musica, 1. Rome: American Institute of Musicology, 1950.

SZENDREI, Janka (Hrsg.). The Istanbul Antiphonal about 1360. Musicalia Danubiana, Vol. 18. Budapest: Akadémiai Kiadó, 2002.

VAN DE KLUNDERT, Sieglinde. Guido von Saint-Denis. Tractatus de tonis. Edition und Studien. (2 vols.) Erlangen: Huricane Publishers, 1998.

\section{Literature}

ATKINSON, Charles M. Parapter. In Handwörterbuch der musikalischen Terminologie, IV. Riethmüller, Albrecht (Hrsg.). Stuttgart: Franz Steiner Verlag, 1978.

ATKINSON, Charles M. The Parapteres: Nothi or Not? Musical Quarterly, 68. 1982, 32-59.

BERNHARD, Michael. The Seligenstadt Tonary. Plainsong and Medieval Music, 13. 2004, S. 107125.

BERNHARD, Michael (Hrsg.). Lexicon Musicum Latitnum Medii Aevi. Wörterbuch der lateinischen Musikterminologie des Mittelalters bis zum Ausgang des 15. Jahrhunderts, 1. Faszikel, Quellenverzeichnis (2. Auflage). München: Verlag der Bayerischen Akademie der Wissenschaften, 2006.

BERNHARD, Michael - WITKOWSKA-ZAREMBA, Elżbieta (Hrsg.). Traditio Iohannis Hollandrini, Band I, Die Lehrtradition des Johannes Hollandrinus. Bayerische Akademie der Wissenschaften. Veröffentlichungen der Musikhistorischen Komission, Band 19. München: Verlag der Bayerischen Akademie der Wissenschaften, 2010.

CLAIRE, Dom Jean. Lés Répertoires Liturgiques Latins Avant Octoechos. Études Grégoriennes, 15. Solesmes: Abbaye Saint-Pierre, 1975. 
CLAIRE, Dom Jean. The Tonus Peregrinus - A Question Well Put? In Orbis Musicae: Studies in Musicology, 7. Tel Aviv: Department of Musicology, Tel Aviv University, 1980, S. 3-15.

DOBSZAY, László - SZENDREI, Janka (Hrsg.). Monumenta Monodica Medii Aevi, Band V, Antiphonen. (3 vols.) Kassel etc.: Bärenreiter, 1999.

DOBSZAY, László. Corpus antiphonarum. Európai örökség és hazai alakítás [Europäisches Erbe und heimische Gestaltung]. Budapest: Balassi Kiadó, 2003.

ERBACHER, Rhabanus. Tonus Peregrinus. Aus der Geschichte eines Psalmtons. Münsterschwarzach: Vier-Türme-Verlag, 1971.

FALCONER, Keith. The Modes before the Modes: Antiphon and Differentia in Western Chant. In The Study of Medieval Chant. Paths and Bridges, East and West. In Honour of Kenneth Levy. Peter Jeffery (ed.). Woodbridge: The Boydell Press, 2001, S. 131-145.

FRERE, Walter Howard. The Use of Sarum, Vol. II. The Ordinal and Tonal. Cambridge: University Press, 1901

FRERE, Walter Howard. Antiphonale Sarisburiense, Vol I. Introduction and Indices. Reprint. Farnborough: Gregg Press, 1966.

HUGLO, Michel. Les Tonaires. Inventaire, Analyse, Comparaison. Paris: Société Française de Musicologie, 1971.

HUGLO, Michel. Grundlagen und Ansätze der mittelalterlichen Musiktheorie von der Spätantike bis zur Ottonischen Zeit. In: Die Lehre vom einstimmigen liturgischen Gesang. Geschichte der Musiktheorie, Bd. 4. Thomas Ertelt - Frieder Zaminer (Hrsg.). Darmstadt: Wissenschaftliche Buchgesellschaft, 2000, S. 17-102.

LIPPHARDT, Walther. Der karolingische Tonar von Metz. Liturgiewissenschaftliche Quellen und Forschungen, 43. Münster: Aschendorffsche Verlagsbuchhandlung, 1965.

LUNDBERG, Mattias. Tonus Peregrinus: The History of a Psalm-tone and its use in Polyphonic Music. Ashgate, 2011.

OMLIN, Ephrem. Die sanktgallischen Tonarbuchstaben. Veröffentlichungen der Gregor. Akademie zu Freiburg (Schweiz). Fellerer, K. G. (Hrsg.), Heft 18. Regensburg : Verlag Friedrich Pustet, 1934.

SEAY, Albert (Hrsg.). Johannes de Olomons (15th Century). Palma Choralis. Colorado Springs: Colorado College Music Press, 1977

STEINER, Ruth. Antiphons for Benedicite at Lauds. Journal of the Plainsong and Mediaeval Music Society 5, 1984, S. 1-17.

SZENDREI, Janka - DOBSZAY, László - RAJECZKY, Benjamin. XVI-XVII. századi dallamaink a népi emlékezetben [Melodien aus dem 16.-17. Jahrhundert in der mündlichen Tradition des Laienvolkes]. (2 vols.) Budapest: Akadémiai Kiadó, 1979. 